Note

\section{Antioxidative Activity of Flavonoids from Thyme (Thymus vulgaris L.)}

\author{
Kayoko MIURA and Nobuji NAKATANI* \\ Department of Food and Nutrition, Faculty of Science \\ of Living, Osaka City University, Sugimoto, \\ Sumiyoshi-ku, Osaka 558, Japan
}

Received April 24, 1989

During the course of our study on the isolation and structural elucidation of antioxidants from the spices of the family Labiatae, we found that the diterpenoids from rosemary ${ }^{1,2)}$ and sage, ${ }^{3)}$ and phenolic acid from oregan $0^{4)}$ had effective antioxidative activity. Recently, we have reported new antioxidative and deodorant biphenyls from thyme (Thymus vulgaris L.). ${ }^{5 \sim 7)}$ This paper deals with the isolation, structural elucidation and antioxidative effect of flavonoids from thyme.

The weakly acidic fraction of the non-volatile part was subjected to column chromatography on silica gel, eluting with benzene-acetone mixture to separate eleven fractions, all of which showed antioxidative activity as strong as that of tert-butyl-4-hydroxytoluene (BHT), one of the synthetic antioxidants. ${ }^{5}$ Each fraction was further purified by silica gel and Sephadex LH-20 column chromatography to afford six flavonoids (flavones $1 \sim 6$ ).<smiles>[R6]c1ccc(-c2cc(=O)c3c(O)c([R6])c([2H])c(Br)c3o2)cc1</smiles>

$\begin{array}{lccccc} & \mathrm{R}_{1} & \mathrm{R}_{2} & \mathrm{R}_{3} & \mathrm{R}_{4} & \mathrm{R}_{5} \\ \text { Flavone 1 } & \mathrm{OCH}_{3} & \mathrm{OCH}_{3} & \mathrm{OCH}_{3} & \mathrm{H} & \mathrm{OH} \\ \text { Flavone 2 } & \mathrm{OCH}_{3} & \mathrm{OCH}_{3} & \mathrm{OCH}_{3} & \mathrm{OCH}_{3} & \mathrm{OH} \\ \text { Flavone 3 } & \mathrm{OCH}_{3} & \mathrm{OCH}_{3} & \mathrm{H} & \mathrm{H} & \mathrm{OH} \\ \text { Flavone 4 } & \mathrm{OCH}_{3} & \mathrm{OCH}_{3} & \mathrm{H} & \mathrm{OCH}_{3} & \mathrm{OH} \\ \text { Flavone 5 } & \mathrm{H} & \mathrm{OCH}_{3} & \mathrm{H} & \mathrm{H} & \mathrm{OH} \\ \text { Flavone 6 } & \mathrm{H} & \mathrm{OCH}_{3} & \mathrm{H} & \mathrm{H} & \mathrm{OCH}_{3}\end{array}$

Flavone 1 was obtained as yellow needles, mp $228^{\circ} \mathrm{C}$, $m / z 344$, from the third fraction. This was identified as 5,4'-dihydroxy-6,7,8-trimethoxyflavone by a comparison with the reported data. ${ }^{8)}$ From the same fraction, flavone 2 was obtained as yellow needles, $\mathrm{mp} 165^{\circ} \mathrm{C}$. The molecular ion peak was observed at $m / z 374$, suggesting it contained one more methoxy group in the B-ring than in that of flavone 1. This compound was identified as $5,4^{\prime}$-dihydroxy-6,7,8,3'-tetramethoxyflavone. ${ }^{9)}$ From the fourth fraction, flavone 3 was obtained as pale yellow needles, $\mathrm{mp} 256^{\circ} \mathrm{C}, \mathrm{m} / \mathrm{z}$ 314. Acetylation of 3 led to a diacetate, whose spectral data were in good agreement with those of the diacetate of 5,4'-dihydroxy-6,7-dimethoxyflavone (cirsilineol) ${ }^{8,9)}$ This supported the identification of flavone 3 as cirsilineol. From the fourth fraction, flavone 4 was also obtained as pale yellow crystals, mp $205^{\circ} \mathrm{C}$. Its spectral characteristics were also similar to those of flavone 2 , except it lacked one methoxy group at the C- 8 position. This compound was identified as 5,4'-dihydroxy-6,7,3'trimethoxyflavone on the basis of spectral data. ${ }^{107}$ The third fraction was triturated with benzene-acetone $(9: 1$, $\mathrm{v} / \mathrm{v}$ ) to separate an insoluble compound, which was recrystallized from acetone to afford a yellow powder, flavone $5, \mathrm{mp} 270^{\circ} \mathrm{C}, \mathrm{m} / \mathrm{z} 284$. Acetylation of 5 led to the diacetate of 5,4'-dihydroxy-7-methoxyflavone (genkwanin). This structure was also supported by the reported spectral data. ${ }^{11)}$ From the first fraction, flavone 6 was

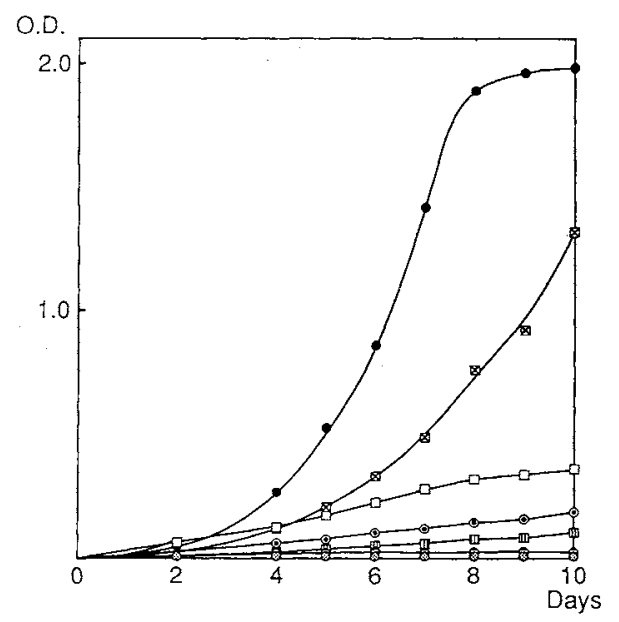

Fig. 1. Antioxidative Activity of Flavonoids from Thyme.

Ferric thiocyanate method, $0.02 \%$ concentration: $\otimes$, flavone $1 ;$, flavone $2 ; \odot$, flavone $4 ; \ominus$, flavone $5 ; \square, \alpha$ tocopherol; $\odot$, BHT; 0 , control.

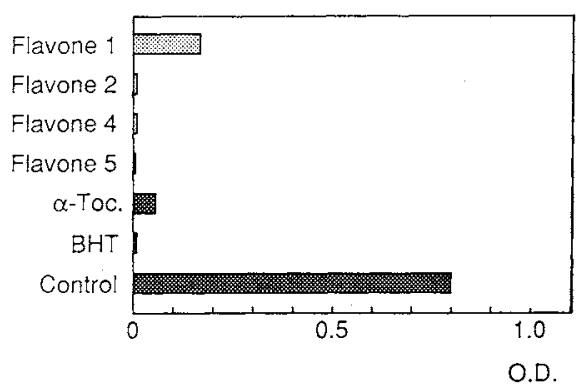

Fig. 2. Antioxidative Activity on the 10th Day of Flavonoids from Thyme (TBA)

* To whom correspondence should be addressed. 
obtained as yellow crystals, mp $173^{\circ} \mathrm{C}$. The ${ }^{1} \mathrm{H}-\mathrm{NMR}$ spectrum of 6 was also similar to that of flavone 5 , except for the presence of a methoxy group on the B-ring instead of the hydroxy group in flavone 5. This compound was identified as 5-hydroxy-7,4'-dimethoxy flavone. ${ }^{811}$ Flavones 1, 4, 5 and 6 were isolated from thyme for the first time.

The antioxidative activity of flavones 1, 2, 4 and 5 was evaluated by the ferric thiocyanate and thiobarbituric acid (TBA) methods at the concentration of $0.02 \%$ as previously reported. ${ }^{11}$ The activity of the flavones 3 and 6 could not be measured because of their low yield. As shown in Fig. 1, the activity of flavones 2,4 and 5 was found to be more than that of $\alpha$-tocopherol, and about the same as that of BHT. Similar results were obtained from the TBA method on the tenth day (Fig. 2). Flavones 2, 4 and $\mathbf{5}$ showed antioxidative activity as high as that of BHT

\section{Experimental}

${ }^{1} \mathrm{H}-\mathrm{NMR}(400 \mathrm{MHz})$ and ${ }^{13} \mathrm{C}-\mathrm{NMR}(100 \mathrm{MHz})$ spectra were run on a JEOL GX-400, using TMS as an internal standerd. MS data were obtained on a Hitachi M-2000.

Extraction and isolation. After removing the non-polar components with $n$-hexane, the leaves $(2 \mathrm{~kg})$ of thyme was extracted with acetone. The acetone extract $(100 \mathrm{~g})$ was steam-distilled and fractionated as descrived in the previous paper. ${ }^{6.7)}$

Flavone 1 (5,4'-dihydroxy-6,7,8-trimethoxy flavone) Yellow needies $(11.12 \mathrm{mg}), \mathrm{mp} 228^{\circ} \mathrm{C}$ (recrystallized from $\mathrm{MeOH})$. MS $m / z(\%): 344.0905\left(\mathrm{M}^{+}, 63\right), 329(100), 211$ (15), $183(17), 118$ (11). UV $\lambda_{\max }(\mathrm{MeOH}) \mathrm{nm}: 232,281$, $294 \mathrm{sh}$. IR $v_{\max }$ (nujol) $\mathrm{cm}^{-1}: 3250,1655,1605,1282,1218$, $1178,1120,1073 .{ }^{1} \mathrm{H}-\mathrm{NMR}$ (acetone- $d_{6}$ ) $\delta: 3.88,3.98,4.08$ ( $3 \mathrm{H}$ each, s), $6.71(1 \mathrm{H}, \mathrm{s}), 7.06(2 \mathrm{H}, \mathrm{dd}, J=1.95,8.79 \mathrm{~Hz})$, $8.00(2 \mathrm{H}, \mathrm{dd}, J=1.95,8.79 \mathrm{~Hz}), 12.80\left(1 \mathrm{H}, \mathrm{s}, \mathrm{D}_{2} \mathrm{O}\right.$ exchangeable).

Flavone 2 (5,4'-dihydroxy-6,7,8,3'-tetramethoxyflavone). Yellow needles $(14.9 \mathrm{mg}), \mathrm{mp} 165^{\circ} \mathrm{C}$ (isopropyl alcohol) MS $m / z(\%): 374\left(\mathrm{M}^{+}, 66\right), 359(100), 211$ (13), 183 (12), 151 (4), 148 (3). UV $i_{\max }(\mathrm{MeOH}) \mathrm{nm}: 253,281,344$. IR $v_{\max }$ (nujol) $\mathrm{cm}^{-1}: 3320,1645,1620,1280,1225,1200 .{ }^{1} \mathrm{H}-$ NMR $\left(\mathrm{CDCl}_{3}\right) \delta: 3.96,3.98,4.00,4.12($ each $3 \mathrm{H}, \mathrm{s}), 6.19$ $\left(1 \mathrm{H}, \mathrm{s}, \mathrm{D}_{2} \mathrm{O}\right.$ exchangeable $), 6.59(1 \mathrm{H}, \mathrm{s}), 7.05(\mathrm{IH}, \mathrm{d}, J=$ $8.30 \mathrm{~Hz}), 7.41(1 \mathrm{H}, \mathrm{d}, J=1.95 \mathrm{~Hz}), 7.54(1 \mathrm{H}, \mathrm{dd}, J=1.95$, $8.30 \mathrm{~Hz}), 12.55\left(1 \mathrm{H}, \mathrm{s}, \mathrm{D}_{2} \mathrm{O}\right.$ exchangeable). ${ }^{13} \mathrm{C}-\mathrm{NMR}$ $\left(\mathrm{CDCl}_{3}\right) \delta: 56.03,61.09,61.69,62.04,103.77,103.80$, $106.95,108.34,115.12,120.76,123.25,132.94,136.58$, $145.73,146.94,149.48,152.97,164.00,182.96$

Flavone 3 (5,4'-dihydroxy-6,7-dimethoxyflavone). Yellow needles $(39 \mathrm{mg}), \mathrm{mp} 256^{\circ} \mathrm{C}$ (acetone). MS $\mathrm{m} / \mathrm{z}(\%): 314$ $\left(\mathrm{M}^{+}, 100\right), 299(70), 284(11), 271(51), 181(30), 121(19)$.
UV $\lambda_{\max }(\mathrm{MeOH}) \mathrm{nm}: 208,284,334$. IR $v_{\max }($ nujol $) \mathrm{cm}^{-1}$ : $3230,1650,1630,1595,1240,1200,1178,1119 .{ }^{1} \mathrm{H}-\mathrm{NMR}$ (acetone- $\left.d_{6}\right) \delta: 3.80,3.99(3 \mathrm{H}$ each, s), $6.68(1 \mathrm{H}, \mathrm{s}), 6.85$ $(1 \mathrm{H}, \mathrm{s}), 7.03(2 \mathrm{H}, \mathrm{d}, J=8.79 \mathrm{~Hz}), 7.96(2 \mathrm{H}, \mathrm{d}, J=8.79 \mathrm{~Hz})$, $9.25\left(1 \mathrm{H}, \mathrm{s}, \mathrm{D}_{2} \mathrm{O}\right.$ exchangeable $), 12.96\left(1 \mathrm{H}, \mathrm{s}, \mathrm{D}_{2} \mathrm{O}\right.$ exchangeable).

Diacetate of flavone 3. IR $v_{\max }$ (film) $\mathrm{cm}^{-1}: 1755,1635$, $1610,1290,1260,1165,1110,1089 .{ }^{1} \mathrm{H}-\mathrm{NMR}\left(\mathrm{CDCl}_{3}\right) \delta$ : $2.35(3 \mathrm{H}, \mathrm{s}), 2.49(3 \mathrm{H}, \mathrm{s}), 3.87,4.00(3 \mathrm{H}$, each s$), 6.58(1 \mathrm{H}$, s), $6.92(1 \mathrm{H}, \mathrm{s}), 7.25(2 \mathrm{H}, \mathrm{d}, J=9.03 \mathrm{~Hz}), 7.88(2 \mathrm{H}, \mathrm{d}, J=$ $9.03 \mathrm{~Hz})$.

Flavone 4 (5,4'-dihydroxy-6,7,3'-trimethoxyflavone). Pale yellow crystals, $m p 205^{\circ} \mathrm{C}(\mathrm{MeOH})$. MS $m / z(\%): 344$ $\left(\mathrm{M}^{+}, 100\right), 329,315,314,181,153,151,149$. UV $\lambda_{\max }$ $(\mathrm{MeOH}) \mathrm{nm}: 274,340$. IR $v_{\max }$ (nujol) $\mathrm{cm}^{-1}: 3250,1655$, $1600,1280,1265,1215,1130 .{ }^{1} \mathrm{H}-\mathrm{NMR}$ (acetone- $d_{6}$ ) $\delta$ : $3.78,3.97,3.98(3 \mathrm{H}$ each, s), $6.73(1 \mathrm{H}, \mathrm{s}), 6.84(1 \mathrm{H}, \mathrm{s}), 7.00$ $(1 \mathrm{H}, \mathrm{d}, J=8.30 \mathrm{~Hz}), 7.62(1 \mathrm{H}, \mathrm{dd}, J=1.95,8.30 \mathrm{~Hz}), 7.64$ $(1 \mathrm{H}, \mathrm{d}, J=1.95 \mathrm{~Hz}), 8.53\left(1 \mathrm{H}, \mathrm{s}, \mathrm{D}_{2} \mathrm{O}\right.$ exchangeable $), 12.96$ $\left(1 \mathrm{H}, \mathrm{s}, \mathrm{D}_{2} \mathrm{O}\right.$ exchangeable). ${ }^{13} \mathrm{C}-\mathrm{NMR}$ (acetone- $\left.d_{6}\right) \delta$ : $56.64,56.77,60.49,91.92,104.22,106.50,110.59,115.58$ $116.38,121.39,123.57,133.51,148.88,151.51,153.95$, $160.06,165.20,183.52$.

Flavone 5 (5,4'dihydroxy-7-methoxyflavone). Yellow amorphous powder $\left(86 \mathrm{mg}\right.$ ), $\mathrm{mp} 270^{\circ} \mathrm{C}$ (acetone). MS $\mathrm{m} / \mathrm{z}$ $(\%): 284\left(\mathrm{M}^{+}, 100\right), 255(36), 241$ (16), 167 (13), $166(14)$, $138(14), 128(16), 121(7), 118(10)$. UV $\lambda_{\max }(\mathrm{MeOH}) \mathrm{nm}$ : $271,300,328$. IR $v_{\max }$ (nujol) $\mathrm{cm}^{-1}: 3220,1660,1600$, $1290,1215,1175 .{ }^{1} \mathrm{H}-\mathrm{NMR}$ (DMSO-d $) \delta: 3.89(3 \mathrm{H}, \mathrm{s})$, $6.40(1 \mathrm{H}, \mathrm{d}, J=2.3 \mathrm{~Hz}), 6.79(1 \mathrm{H}, \mathrm{d}, J=2.3 \mathrm{~Hz}), 6.85(1 \mathrm{H}$, s), $6.96(2 \mathrm{H}, \mathrm{d}, J=9.3 \mathrm{~Hz}), 7.98(2 \mathrm{H}, \mathrm{d}, J=9.3 \mathrm{~Hz}), 12.95$ ( $1 \mathrm{H}, \mathrm{s}, \mathrm{D}_{2} \mathrm{O}$ exchangeable).

Diacetate of flavone 5. MS $m / z(\%): 368\left(\mathrm{M}^{+}, 3\right), 326$ (53), $284(100), 255(22)$. IR $v_{\max }$ (nujol) $\mathrm{cm}^{-1}: 1765,1750$, $1638,1610,1580,1295,1225,1210,1195,1173,1110 .{ }^{1} \mathrm{H}-$ NMR $\left(\mathrm{CDCl}_{3}\right) \delta: 2.27(3 \mathrm{H}, \mathrm{s}), 2.36(3 \mathrm{H}, \mathrm{s}), 3.85(3 \mathrm{H}, \mathrm{s})$, $6.52(1 \mathrm{H}, \mathrm{s}), 6.58(1 \mathrm{H}, \mathrm{d}, J=2.4 \mathrm{~Hz}), 6.82(1 \mathrm{H}, \mathrm{d}, J=$ $2.4 \mathrm{~Hz}), 7.20(1 \mathrm{H}, \mathrm{dd}, J=2.4,8.4 \mathrm{~Hz}), 7.84(1 \mathrm{H}, \mathrm{dd}, J=$ $2.4,8.4 \mathrm{~Hz})$.

Flavone 6 (5-hydroxy-7,4'dimethoxyflavone). Yellow crystals $(2.17 \mathrm{mg}), \mathrm{mp} 173^{\circ} \mathrm{C}$. (acetone) MS m/z: 298, 269, 255, 166, 138, 135, 132. UV $\lambda_{\max }(\mathrm{MeOH}): 273,301,324$. IR $v_{\max }$ (nujol) $\mathrm{cm}^{-1}: 1665,1605,1585,1270,1165 .{ }^{1} \mathrm{H}-$ NMR $\left(\mathrm{CDCl}_{3}\right) \delta: 3.89,3.90(3 \mathrm{H}$ each, $s), 6.37(1 \mathrm{H}, \mathrm{d}, J=$ $2.4 \mathrm{~Hz}), 6.49(1 \mathrm{H}, \mathrm{d}, J=2.4 \mathrm{~Hz}), 6.58(1 \mathrm{H}, \mathrm{s}), 7.02(2 \mathrm{H}, \mathrm{d}$, $J=9.3 \mathrm{~Hz}), 7.85(2 \mathrm{H}, \mathrm{d}, J=9.3 \mathrm{~Hz}), 12.80(1 \mathrm{H}, \mathrm{s})$

\section{References}

1) R. Inatani, N. Nakatani and H. Fuwa, Agric. Biol. Chem., 47, 521 (1983) 
2) N. Nakatani and R. Inatani, Agric. Biol. Chem., 48, 2081 (1984).

3) N. Nakatani, H. Kikuzaki and R. Inatani, Abstracts of Papers, Annual Meeting of the Japanese Society of Nutrition and Food Science, Osaka, May 1983, p. 4.

4) H. Kikuzaki and N. Nakatani, Agric. Biol. Chem., 53, 519 (1989).

5) K. Miura and N. Nakatani, Chem. Express, 4, 237 (1989).

6) N. Nakatani, K. Miura and T. Inagaki, Agric. Biol. Chem., 53, 1375 (1989).

7) K. Miura, T. Inagaki and N. Nakatani, Chem.
Pharm. Bull., 37, 1816 (1989).

8) A. G. Gonzalez, B. M. Fraga, M. G. Hernandez, F. Larruga, J. G. Luis and A. G. Ravelo, Lloydia, 41, 279 (1978).

9) C. O. Von Den Broucke, R. A. Dommisse, E. L. Esman and J. A. Lemli, Phytochemistry, 21, 2581 (1982).

10) N. Morita, M. Shimizu and M. Arisawa, Phytochemistry, 12, 421 (1973).

11) C. H. Brieskorn and H. Michel, Tetrahedron Lett., 30, 3447 (1968). 